\title{
Étude phénotypique de la résistance des bactéries isolées des eaux des lacs et rivières de la ville de Franceville aux céphalosporines de troisième génération
}

\author{
Jean-Fabrice YALA ${ }^{1 *}$, Rolande MABIKA MABIKA ${ }^{1}$, Danielle BOUCKA BOUCKA ${ }^{1}$, Alexis Nicaise \\ LEPENGUE2, Alain SOUZA' ${ }^{1}$. \\ 1. Laboratoire de Biologie Moléculaire et Cellulaire; équipe de Microbiologie, Unité de recherche Agrobiologie, \\ Université des Sciences et Techniques de Masuku (USTM), BP 067 Franceville, Gabon. \\ 2. Laboratoire de Physiologie végétale, Phytopathologie et Amélioration des plantes, Unité de recherche \\ Agrobiologie, Université des Sciences et Techniques de Masuku (USTM), BP 067 Franceville, Gabon \\ ${ }^{*}$ Corresponding Author : Jean-Fabrice YALA \\ Laboratoire de Biologie Moléculaire et Cellulaire ; Équipe Microbiologie et Immunologie, Université des Sciences et \\ Techniques de Masuku (USTM), BP 067 Franceville, Gabon. Correspondant E. mail : (yalalaw@yahoo.fr) Tel : \\ 04.35.91.02/06.75.46.87
}

Original submitted in on 26 th September 2017. Published online at www.m.elewa.org on 30th November 2017 https://dx.doi.org/10.4314/jab.v119i1.12

\section{RÉSUMÉ}

Objectif: Cette étude explore la sensibilité des bactéries Gram- de forme bacillaire (Aeromonas salmonicida, Escherichia coli 1, Escherichia coli 2, Klebsiella pneumoniae rhinosclero, Pseudomonas aeruginosa et Yersinia enterocolitica) isolées des eaux des lacs, de la rivière M'passa et du fleuve Ogooué au Gabon aux céphalosporines de troisième génération (ceftriaxone, céfotaxime, cefpodoxime et céfixime).

Méthodologie et résultats: Ces bactéries ont d'abords été identifiées par le système Api $20 \mathrm{E}$, puis soumises à l'antibiogramme pour évaluer leur sensibilité aux quatre (4) antibiotiques. De ce fait, la sensibilité des bactéries aux céphalosporines de troisième génération a été évaluée par la méthode de puits dans la gélose Mueller Hinton. Le diamètre de la zone d'inhibition autour de chaque puit obtenu après 18-24 heures d'incubation a été mesuré à l'aide d'un pied à coulisse. Au regard des résultats de cette étude, deux comportements majeurs des souches bactériennes testées se dégagent, d'une part la sensibilité et d'autre part la multirésistance observée avec la plupart des antibiotiques. Effectivement, Escherichia coli1, Escherichia coli2, Klebsiella pneumoniae rhinosclero, Yersinia enterocolitica sont sensibles à la ceftriaxone et à la céfotaxime. Par contre, la multirésistance est observée avec Pseudomonas aeruginosa, Aeromonas salmonicida et Escherichia coli du milieu hospitalier qui présente toutes un caractère de résistante aux quatre (4) antibiotiques.

Conclusion et application des résultats : En somme, le phénomène de multirésistance est présent chez les bactéries isolées des eaux de Franceville. Donc, il est important de mettre en place un système de surveillance et de suivi de la qualité microbiologique de ces eaux.

Mots clés : Antibiotiques, bactéries, multirésistance, sensibilité, céphalosporine. 


\section{ABSTRACT}

Objective :This study investigates the susceptibility of Gram- bacteria (Aeromonas salmonicida, Escherichia coli 1, Escherichia coli 2, Klebsiella pneumoniae rhinosclero, Pseudomonas aeruginosa and Yersinia enterocolitica) to third generation cephalosporins (ceftriaxone, cefotaxime, cefpodoxime and cefixime) using water samples collected from the lakes, the M'passa and the Ogooué Rivers in Gbon.

Methodology and Results: The bacteria were first re-identified by the Api $20 \mathrm{E}$ system, and then subjected to the antibiogram to evaluate of their sensibilities to four (4) antibiotics, namely ceftriaxone, cefotaxime, cefpodoxime and cefixime. Therefore, the susceptibility of bacteria to the third generation cephalosporins was evaluated by the well method in Mueller Hinton agar. The diameter of the inhibition zone around each well was obtained after 18-24 hours of incubation and measured by means of a caliper. The results of this study revealed that the tested bacterial strains fall into two major groups, the sensible and the multiresistance ones. Indeed, Escherichia coli 1, Escherichia coli 2, Klebsiella pneumoniae rhinosclero, Yersinia enterocolitica were susceptible to ceftriaxone and cefotaxime. While, the multiresistance was observed with Pseudomonas aeruginosa, Aeromonas salmonicida and Escherichia coli of the hospital who presents quite a character of resistant to four (4) antibiotics.

Conclusion and application of results: In summary, the phenomenon of multiresistance was observed in the bacteria isolated from the waters samples of Franceville. Therefore, it is important to set up a system for monitoring and monitoring the microbiological quality of these waters.

Keywords : Antibiotics, bacteria, multiresistance, susceptibility, cephalosporin.

\section{INTRODUCTION}

Ces dernières années, plusieurs études ont mis en évidence la présence de souches d'Entérobactéries productrices des $\beta$-lactamases à Spectre Etendu (EBLSE) dans les effluents hospitaliers, aussi bien dans des pays en voie de développement que dans des pays développés. En effet, Galvin et al se sont intéressés à l'impact des effluents hospitaliers en quantifiant les souches d'Escherichia coli (E. coli) et les souches d'E. coli résistantes aux antibiotiques dans des échantillons d'effluents prélevés à différents niveaux. II ressort de façon évidente que parmi les souches résistantes, des EBLSE ont été détectées et caractérisées, et portent des gènes codant pour une $\beta$-lactamase à Spectre Etendu (BLSE) de type CTX-M du groupe CTXM-1 (Galvin et al . 2010). De même, des souches de Klebsiella pneumoniae (K. pneumoniae) et $E$. coli ont été détectées dans les effluents hospitaliers, dans les boues activées et dans les effluents d'une station d'épuration (Prado et al . 2008; Harris et al . 2014). Ainsi, les rejets des déchets hospitaliers, industriels, agricoles, domestiques, des activités vétérinaires et les bactéries fécales dans l'environnement conduisent systématiquement à la contamination des milieux aquatiques naturels (Garcia-Armisen et al . 2013; Garcia-Armisen et al . 2006; GarciaArmisen et al . 2011; Sidrach-Cardona et al . 2014). De plus, les antibiotiques et leurs métabolites relargués dans l'environnement ont un impact direct sur les microorganismes de l'environnement. L'impact de ces molécules sur les microorganismes a un regain d'attention comparable à celui des polluants ces dernières années (Costanzo et al . 2005; Hijosa-Valsero et al 2011; Sidrach-Cardona and Bécares 2013; Sidrach-Cardona et al . 2014). Effectivement, plusieurs travaux d'une part axés sur l'analyse des concentrations des antibiotiques dans les milieux aquatiques a montré la présence des différentes familles d'antibiotiques dont les concentrations pouvant atteindre $37,3 \mu \mathrm{g} . \mathrm{L}^{-1}$ pour la vancomycine (Passerat et al . 2010). D'autre part, l'étude des profils de résistance des bactéries isolées des milieux aquatiques. Ces études soulignent la résistance d'E. coli, des coliformes totaux, des entérocoques à l'amoxicilline, à l'azithromycine, la pénicilline, à l'érythromycine (Sidrach-Cardona and Bécares 2013; Sidrach-Cardona et al . 2014).

Aussi, la présence de ces molécules a pour conséquence l'augmentation de la pression sélective sur les bactéries, favorise leur résistance, 
lesquelles réduisent le potentiel thérapeutique (Hijosa-Valsero et al . 2011). En revanche, la présence des pathogènes entériques dans les environnements aquatiques peut occasionner des maladies avec des risques élevés lorsque les bactéries sont résistantes aux antibiotiques (Servais and Passerat 2009). Les eaux de surface sont les réceptacles des eaux usées aussi bien industrielles, agricoles, hospitalières que domestiques. Ces eaux de surface polluées, dans certains pays peuvent conduire à la contamination des eaux de consommation, à la contamination directe des personnes qui les consomment. Les eaux de surface étant également utilisées pour l'arrosage des cultures, l'homme peut être contaminé indirectement en ingérant des végétaux contaminés. Toutefois, au Gabon, les données de la contamination de l'environnement par les entérobactéries ou les bactéries Gram- résistantes aux antimicrobiens ne sont pas connues. Par

\section{MATERIELS ET METHODES}

Cette étude a porté sur quelques bactéries Gramisolées des eaux des lacs Makana et Lacaisse, de la rivière M'passa et du fleuve Ogooué au laboratoire de Biologie Moléculaire et Cellulaire du Département de Biologie et qui ont été conservé à $-20^{\circ} \mathrm{C}$. Ces bactéries ont été isolées en 2013.

\section{Identification des bactéries isolées}

Préparation des cultures de la nuit et isolement des colonies : A partir des cultures cryoconservées, $50 \mu \mathrm{l}$ ont été mis dans $900 \mu \mathrm{l}$ de milieu liquide BrainHeart Infusion (BHI) et le tout incubé à $37^{\circ} \mathrm{C}$ pendant 18-24 heures. A partir de la culture de la nuit, $10 \mu$ l ont été prélevés et ensemencés par la méthode de stries sur la gélose $\mathrm{BHI}$. Enfin, les boites ont été incubées à $37^{\circ} \mathrm{C}$ pendant 18 à 24 heures.

Identification biochimique des souches bactériennes : Une colonie isolée a été délayée dans $3 \mathrm{ml}$ de sérum physiologique $(0,9 \% \mathrm{NaCl})$ jusqu'à l'obtention d'une opacité de 0,5 Mac Farland correspondant à $10^{5}-10^{6}$ cellules $/ \mathrm{ml}$. Ensuite, chaque cupule a été inoculée avec l'inoculum. Enfin, les galeries ont été incubées à $37^{\circ} \mathrm{C}$ pendant $18-24$ heures. L'identification des souches a été faite à l'aide du catalogue Api $20 \mathrm{E}$.

L'antibiogramme : Dans cette étude, l'antibiogramme nous a permis d'avoir des indications sur l'efficacité in vitro des céphalosporines de ailleurs, dans la ville de Franceville, les eaux usées, les déchets des usines et des hôpitaux sont directement mis en contact avec les écosystèmes aquatiques : lacs, rivières, fleuves et étangs. Ainsi, il nous a semblé opportun d'évaluer la sensibilité des bactéries isolées des eaux de la ville de Franceville aux céphalosporines de troisième génération. Manifestement, les $\beta$-lactamines représentent la principale famille d'antibiotiques la plus développée et la plus utilisée dans le monde. Cette large utilisation est due à leur large spectre d'activités antibactériennes, leur faible toxicité, leur efficacité et leur faible cout pour certaine molécule (Baba Ahmed-Kazi Tani and Arlet 2014). Le but de ce travail a consisté à identifier à partir du souchier les bactéries Gram- isolées des eaux des lacs, de la rivière M'passa et du fleuve Ogooué, puis d'évaluer in vitro la sensibilité de ces germes ainsi qu'une bactérie isolée du milieu hospitalier à la ceftriaxone, céfotaxime, céfixime et cefpodoxime.

troisième génération à savoir : ceftriaxone, céfotaxime, cefpodoxime et céfixime sur les bactéries Gramisolées des eaux de Franceville mais également à une souche d'E. coli isolée du milieu hospitalier.

A partir d'une culture de la nuit, un inoculum de densité optique finale de 0,8 a été préparé.

La gélose Mueller-Hinton (MH) a été ensemencée par inondation puis laissée sécher 10-15 minutes sous la hotte. Ensuite, les puits ont été faits dans la gélose et $50 \mu \mathrm{l}$ d'antibiotique de concentration $30 \mu \mathrm{g} / \mathrm{ml}$ ont été déposés. Les boites de pétries ont été incubée à $37^{\circ} \mathrm{C}$ pendant 18-24h. Enfin, les diamètres d'inhibition obtenus après la période d'incubation ont été mesurés à l'aide du pied à coulisse.

Détermination de la concentration minimale inhibitrice : CMI

La méthode de microdilution en milieu liquide $\mathrm{BH}$ dans des microplaques de 96 puits a été utilisée pour évaluer les paramètres d'inhibition de croissance des bactéries par les antibiotiques. En effet, des dilutions géométriques de raison 2 ont été réalisées (de $1 / 2$ à 1/512) à partir d'une solution d'antibiotique de $30 \mu \mathrm{g} / \mathrm{ml}$. La microplaque a été recouverte et incubée à $37^{\circ} \mathrm{C}$ pendant $18-24$ heures. La concentration la plus faible d'antibiotique inhibant toute croissance visible à l'œil nu après la durée d'incubation est la CMI. Ainsi, 
trois essais ont été réalisés pour chacun des germes et la valeur de la CMl est la moyenne des trois tests.

Détermination de la concentration minimale bactéricide : CMB

La concentration minimale bactéricide ou CMB a été déterminée en ensemençant sur le milieu LauriaBertani (LB) par étalement $100 \mu$ l des échantillons des puits ne présentant pas de croissance dans la microplaque. La concentration d'antibiotique la plus faible ayant décimée $99,99 \%$ de la population de départ, après 24 heures d'incubation à $37{ }^{\circ} \mathrm{C}$ correspond à la CMB. L'activité intrinsèque des différents antibiotiques a été déterminée en fonction du rapport $\mathrm{CMB} / \mathrm{CMI}$ nommé $a$. En effet, si $1 \leq a \leq 2$, l'effet est bactéricide et si $4 \leq a \leq 16$, l'effet est bactériostatique. En outre, toute valeur de a supérieure à 16, l'activité est dite tolérante.

Analyse statistique: L'analyse statistique des résultats a été faite à l'aide du logiciel $R$, version 3.0.2 (2013-09-25).

\section{RESULTATS :}

Identification des bactéries isolées

Tableau 1. Résultats de l'identification

\begin{tabular}{c|c|c}
\hline Souches & Codes & Noms \\
\hline E5 & $\mathbf{0 0 0 4 5 3 1 1}$ & Klebsiella pneumoniae rhinosclero \\
\hline E6 & 20020042 & Pseudomonas aeruginosa \\
\hline E17 & $\mathbf{0 0 1 4 1 2 3}$ & Yersinia enterocolitica \\
\hline F15 & 2002004 & Aeromonas salmonicida \\
\hline F43 & 1044542 & Escherichia coli 2 \\
\hline F46 & 1044502 & Escherichia coli 1 \\
\hline
\end{tabular}

Ces résultats confirment non seulement la présence des bactéries de la famille des Entérobacteriacea (Escherichia coli 1, Escherichia coli 2, Klebsiella Pneumoniae rhinosclero, Yersinia enterocolitica) mais aussi celles des familles des Pseudomonadaceae
(Pseudomonas aeruginosa) et Aeromonadaceae (Aeromonas salmonicida) dans les eaux des lacs Makana et Lacaisse, de la rivière la M'passa et du fleuve Ogooué (tableau 1).

\section{Antibiogramme}

Tableau 2 : Sensibilité des bactéries aux céphalosporines de $3^{\text {ème }}$ génération.

\begin{tabular}{l|c|c|c|c}
\hline & \multicolumn{4}{|c}{ Diamètres d'inhibition (mm) } \\
\hline Souches & Ceftriaxone & Céfotaxime & Cefpodoxime & Céfixime \\
\hline Escherichia coli1 & $19,83 \pm 1,61^{\mathrm{S}}$ & $19,17 \pm 0,58^{\mathrm{S}}$ & $0 \pm 0^{\mathrm{R}}$ & $14 \pm 0,87^{\mathrm{R}}$ \\
\hline Escherichia coli2 & $20,33 \pm 1,04^{\mathrm{S}}$ & $20,17 \pm 1,04^{\mathrm{S}}$ & $0 \pm 0^{\mathrm{R}}$ & $14,83 \pm 3,33^{\mathrm{R}}$ \\
\hline Klebsiella pneumoniae rhinosclero & $19,58 \pm 0,80^{\mathrm{S}}$ & $20,67 \pm 1,15^{\mathrm{S}}$ & $0 \pm 0^{\mathrm{R}}$ & $15,25 \pm 4,55^{\mathrm{R}}$ \\
\hline Pseudomonas aeruginosa & $0 \pm 0^{\mathrm{R}}$ & $0 \pm 0^{\mathrm{R}}$ & $0 \pm 0^{\mathrm{R}}$ & $0 \pm 0^{\mathrm{R}}$ \\
\hline Aeromonas salmonicida & $0 \pm 0^{\mathrm{R}}$ & $0 \pm 0^{\mathrm{R}}$ & $0 \pm 0^{\mathrm{R}}$ & $12,5 \pm 0,70^{\mathrm{R}}$ \\
\hline Escherichia coli & $13 \pm 1,41^{\mathrm{S}}$ & $14 \pm 0^{\mathrm{R}}$ & $0 \pm 0^{\mathrm{R}}$ & $12,5 \pm 3,53^{\mathrm{R}}$ \\
\hline Yersinia enterocolitica & $19,25 \pm 2,47^{\mathrm{S}}$ & $19,25 \pm 1,76^{\mathrm{S}}$ & $0 \pm 0^{\mathrm{R}}$ & $11,25 \pm 1,76^{\mathrm{R}}$ \\
\hline
\end{tabular}

$\mathrm{S}$ : sensible, $\mathrm{R}$ : résistant

Les résultats du tableau 2 ne révèlent aucun diamètre d'inhibition des six souches bactériennes isolées des eaux des lacs Makana et Lacaisse, des eaux de la M'passa et de l'Ogooué en présence de la cefpodoxime. Aussi, Pseudomanas aeruginosa ( $P$. aeruginosa) ne présente aucun diamètre d'inhibition avec les quatre (4) antibiotiques testés. II en est de même pour Aeromonas salmonicida (A. Salmonicida) bien qu'ayant obtenu un faible diamètre d'inhibition avec la céfixime. Toutefois, les céphalosporines de troisième génération $\left(\mathrm{C}_{3} \mathrm{G}\right)$ qui présent les plus grands diamètres d'inhibition sont la ceftriaxone et la céfotaxime respectivement pour Escherichia coli 2 ( $E$. coli 2) et Klebsiella pneumoniae rhinosclero ( $K$. 
Yala et al., J. Appl. Biosci. 2017 Étude phénotypique de la résistance des bactéries isolées des eaux des lacs et rivières de la ville de Franceville aux céphalosporines de troisième génération

pneumoniae rhinosclero). De plus, les plus petits diamètres sont obtenus avec la céfixime, 12,5 et 11,25 $\mathrm{mm}$ respectivement pour $A$. salmonicida et Yersinia enterocolitica (Y. enterocolitica) (tableau2). Les résultats montrent également que $E$. coli (milieu hospitalier) présente des petits diamètres en présence de la céfotaxime, la céfixime et la cefpodoxime, mais aucun avec la ceftriaxone. Enfin, l'étude statistique réalisée par le test de kruskal-Wallis montre que les différences entre les diamètres d'inhibition ne sont pas significatives car les $p$-value $(0,3528 ; 0,3528$ et 0,3286$)$ sont supérieures au seuil de risque $a=0,05$ respectivement pour la ceftriaxone, la céfotaxime et la céfixime. Sur les six souches testées à la ceftriaxone, quatre d'entre elles se sont révélé être sensibles (E.coli1, E.coli2, K. pneumoniae rhinosclero et $Y$. enterocolitica) et les deux autres résistantes ( $P$. aeruginosa et $A$. salmonicida) (tableau 2). Ces résultats sont également observés avec la céfotaxime. Pour ce qui est des tests en présence de la cefpodoxime et la céfixime, toutes les bactéries isolées de l'environnement se sont révélées résistantes à ces deux antibiotiques. Enfin, la souche provenant du milieu hospitalier $(E$. coli) s'est révélée être résistante aux quatre céphalosporines de troisième génération.

\section{Détermination de la CMI}

Tableau 3. Les différentes valeurs des CMI des céphalosporines de 3ème génération

\begin{tabular}{l|c|c|c|c}
\hline & \multicolumn{3}{|c}{ CMI $(\boldsymbol{\mu g} / \mathrm{ml})$} \\
\hline Souches & Ceftriaxone & Céfotaxime & Cefpodoxime & Céfixime \\
\hline Escherichia coli1 & $\mathbf{2 , 8 1 \pm 1 , 3 2}$ & $\mathbf{5 , 6 2 \pm 2 , 6 5}$ & $30^{*}$ & $15 \pm 0$ \\
\hline Escherichia coli2 & $2,81 \pm 1,32$ & $5,62 \pm 2,65$ & $30^{*}$ & $15 \pm 0$ \\
\hline Klebsiella pneumoniae rhinosclero & $3,75 \pm 0$ & $5,62 \pm 2,65$ & $30^{*}$ & $15 \pm 0$ \\
\hline Pseudomonas aeruginosa & $30^{*}$ & $30^{*}$ & $30^{*}$ & $30^{*}$ \\
\hline Aeromonas salmonicida & $30^{*}$ & $30^{*}$ & $30^{*}$ & $30^{*}$ \\
\hline Escherichia coli & $30^{*}$ & $30^{*}$ & $30^{*}$ & $30^{*}$ \\
\hline Yersinia enterocolitica & $1,87 \pm 0$ & $15 \pm 0$ & $30^{*}$ & $30^{*}$ \\
\hline
\end{tabular}

${ }^{*} \mathrm{CMl}>30 \mu \mathrm{g} / \mathrm{ml}$

L'analyse des résultats du tableau 3 montre que les souches étant toutes résistantes à la cefpodoxime aucune CMI n'a été déterminée dans la gamme de concentration que nous avons utilisée. Aussi, aucune CMI n'a été déterminée avec les quatre (4) antibiotiques pour $P$. aeruginosa, $A$. salmonicida et $E$. coli du milieu hospitalier. Les plus petites CMI sont obtenues avec le ceftriaxone et la céfotaxime respectivement pour $Y$. enterocolitica, $E$. coli1 et $E$. coli2. Enfin, la céfixime montre les plus grandes $\mathrm{CMI}$.

\section{Détermination de la CMB}

Tableau 4. Les différentes valeurs des CMB des céphalosporines de Зème génération

\begin{tabular}{l|c|c|c|c}
\hline & \multicolumn{4}{|c}{ CMB $(\boldsymbol{\mu g} / \mathrm{ml})$} \\
\hline Souches & Ceftriaxone & Céfotaxime & Cefpodoxime & Céfixime \\
\hline Escherichia coli1 & $\mathbf{2 , 8 1 \pm 1 , 3 3}$ & $\mathbf{5 , 6 2 \pm 2 , 6 5}$ & Ind & $15 \pm 0$ \\
\hline Escherichia coli2 & $\mathbf{2 , 8 1 \pm 1 , 3 3}$ & $\mathbf{5 , 6 2 \pm 2 , 6 5}$ & Ind & $15 \pm 0$ \\
\hline Klebsiella pneumoniae rhinosclero & $3.75 \pm 0$ & Ind & Ind & $15 \pm 0$ \\
\hline Pseudomonas aeruginosa & Ind & Ind & Ind & Ind \\
\hline Aeromonas salmonicida & Ind & Ind & Ind & Ind \\
\hline Escherichia coli & Ind & Ind & Ind & Ind \\
\hline Yersinia enterocolitica & $\mathbf{1 , 8 7 \pm 0}$ & $\mathbf{1 5 \pm 0}$ & Ind & Ind \\
\hline
\end{tabular}

Ind : indéterminée 
Yala et al., J. Appl. Biosci. 2017 Étude phénotypique de la résistance des bactéries isolées des eaux des lacs et rivières de la ville de Franceville aux céphalosporines de troisième génération

Le tableau 4 montre qu'aucune CMB n'est déterminée avec la cefpodoxime. Aussi, les plus grandes CMB sont obtenues avec la céfixime et les plus petites avec la ceftriaxone. Enfin, aucune CMB n'est déterminée pour
$P$. aeruginosa, $A$. salmonicida avec les $C_{3} G$ testés. ॥ en est de même pour $E$. coli du milieu hospitalier.

\section{Evaluation du rapport $\mathrm{CMB} / \mathrm{CMI}$}

Tableau 5. Résultats du rapport a

\begin{tabular}{c|c|c|c|c}
\hline & \multicolumn{4}{|c}{ Rapport $\mathbf{a}$} \\
\hline Souches & Ceftriaxone & Céfotaxime & Cefpodoxime & Céfixime \\
\hline Escherichia coli1 & $\mathbf{1 \pm 0}$ & $\mathbf{1 \pm 0}$ & Ind & 1 \\
\hline Escherichia coli2 & $\mathbf{1 \pm 0}$ & $\mathbf{1 \pm 0}$ & Ind & $\mathbf{1}$ \\
\hline Klebsiella pneumoniae rhinosclero & $\mathbf{1 \pm 0}$ & $\mathbf{1 \pm 0}$ & Ind & $\mathbf{1}$ \\
\hline Pseudomonas aeruginosa & Ind & Ind & Ind & Ind \\
\hline Aeromonas salmonicida & Ind & Ind & Ind & Ind \\
\hline Escherichia coli & Ind & Ind & Ind & Ind \\
\hline Yersinia enterocolitica & $\mathbf{1 \pm 0}$ & $\mathbf{1 \pm 0}$ & Ind & Ind \\
\hline
\end{tabular}

Ind : indéterminé

Le rapport a montre que les $\mathrm{CMB}$ sont égales aux $\mathrm{CMI}$ pour les $\mathrm{C}_{3} \mathrm{G}$ testés chez $E$. coli1, $E$. coli2, $K$. pneumoniae rhinosclero et $Y$. enterocolitica (tableau5).

\section{DISCUSSION}

Au terme de cette étude qui s'est proposée d'évaluer la sensibilité des bactéries fécales isolées des eaux des lacs, de la M'passa et de l'Ogooué aux céphalosporines de troisième génération $\left(\mathrm{C}_{3} \mathrm{G}\right)$. L'analyse des résultats de la ré identification confirme la présence des bactéries de différentes familles bactériennes dans ces eaux, qui appartiennent aux bactéries fécales. Ces résultats sont en accord avec l'étude menée par Nola et al qui ont montrés l'abondance de Pseudomonadacea et Aeromonadacea dans les eaux de la zone équatoriale (Nola et al . 2001). La non significativité de la différence des diamètres d'inhibition (tableau2) pourrait se justifier par le fait que d'une part, les quatre (4) céphalosporines de troisième génération appartiendraient à la même famille d'antibiotiques et par conséquent, ils auraient le même mécanisme d'action, et d'autre part, que les souches $K$. pneumoniae rhinosclero, E. coli1, E. coli2, E. coli (milieu hospitalier), $Y$. enterocolitica sont toutes des entérobactéries. L'analyse des résultats de l'évaluation de la sensibilité des bactéries vis-à-vis des $\mathrm{C}_{3} \mathrm{G}$ (tableau2) varie en fonction de la classe des $\mathrm{C}_{3} \mathrm{G}$. Effectivement, les souches isolées de l'environnement sont toutes résistantes à la cefpodoxime et à la céfixime. Cette résistance pourrait s'expliquer par une pression de sélection des bactéries due à la présence
Donc ces antibiotiques sont bactéricides, c'est-à-dire qu'en plus d'inhiber la croissance bactérienne, ces $\mathrm{C}_{3} \mathrm{G}$ détruisent aussi ces souches bactériennes.

des antibiotiques et d'autres molécules dans les effluents hospitaliers, déchets industriels, agricoles et domestiques. En effet, des travaux ont montré que ces déchets étaient directement évacués dans l'environnement, notamment dans les eaux de surfaces ou les eaux souterraines via les égouts favorisant ainsi l'émergence des bactéries multirésistance (Weiss 2002; Drieux-Rouzet and Jarlier 2014; Harris et al . 2014). En outre, les études récentes, menées sur la détection et la quantification des biomolécules dans les effluents et les eaux de rivières ont révélé la présence des antibiotiques à des concentrations variables (Servais and Passerat 2009; Passerat et al . 2010). Ces dernières et leurs métabolites maintiendraient la pression de sélection et augmenteraient le taux des bactéries multirésistantes (Andremont 1997.; Andremont 2002). En définitive, les antibiotiques et autres molécules relarguées dans les milieux aquatiques naturels semblent favoriser ainsi l'émergence des souches bactériennes multirésistantes. Aussi, Drieux et Jarlier ont démontré dans leurs travaux que l'activité humaine a un impact sur les écosystèmes aquatiques en termes de multirésistance aux antibiotiques (Drieux-Rouzet and Jarlier 2014). Par ailleurs, l'échange du matériel génétique pourrait dans tous les cas expliquer cette 
résistance observée chez les bactéries isolées des eaux des lacs, de l'Ogooué et de la M'passa. Manifestement, durant leur transit dans le tube digestif des humains et animaux traités aux antibiotiques, les bactéries fécales peuvent acquérir une certaine résistance aux antibiotiques (Weiss 2002; Servais and Passerat 2009). Par conséquent, les bactéries présentes dans les eaux des milieux naturels peuvent $y$ acquérir une forme d'antibiorésistance par échange de matériel génétique avec ces dernières (Servais and Passerat 2009). De plus, l'échange des informations génétiques entre souche de même espèce justifierait le statut observé. Effectivement, les bacilles à Gram- en général et les entérobactéries en particulier ont cette capacité et facilité d'échanger l'information génétique entres elles ou avec d'autres bactéries de l'environnement (Baba Ahmed-Kazi Tani and Arlet 2014). Ce qui augmenterait la multirésistance vis-à-vis des antibiotiques. Au regard des résultats obtenus, il ressort que les souches de $P$. aeruginosa et $A$. salmonicida sont particulièrement résistantes aux quatre antibiotiques testés à savoir: ceftriaxone, céfotaxime, cefpodoxime et céfixime. Le caractère résistant observé chez ces deux souches bactériennes serait naturel ou acquit. Assurément, plusieurs études ont montré de façon évidente la résistance naturelle chez $P$. aeruginosa (Baba Ahmed-Kazi Tani and Arlet 2014) qui lui permet de résister à plusieurs $\beta$ lactamines par production d'une $\beta$-lactamase chromosomique de classe $\mathrm{C}$ inductible (Philippon et al . 2002; Baba Ahmed-Kazi Tani and Arlet 2014) et, qui ne serait pas inhiber par l'acide clavulanique et hydrolyserait les $\mathrm{C}_{3} \mathrm{G}$ telles que la ceftazidime et la céfépime (Mérens et al . 2011). De plus, les $\beta$ lactamases à spectre élargi (BLSE) constituent un groupe d'enzymes dont la particularité est de conférer la résistance aux céphalosporines de première, deuxième et troisième génération (Bush et al . 1995). En outre, la résistance acquise chez $P$. aeruginosa résulterait certainement d'une modification génétique qui serait due soit à une mutation ou à l'acquisition d'un gène de résistance (Mérens et al . 2011). Indéniablement, la paroi des bacilles Gram- du fait de la présence de la membrane externe est moins perméable (Kulkarni et al . 2015). Par conséquent, la modification de certaines porines en particulier la porine OprD chez $P$. aeruginosa justifierait ce statut (Stratton 1990; Lister et al . 2009; Mérens et al . 2011). Le niveau de résistance élevé d'A. salmonicida s'expliquerait par différents mécanismes de résistance : sécrétion d'enzymes, imperméabilité de la membrane externe et pompe à efflux (Li and Nikaido 2009; Li et al . 2015). Cette multirésistance observée chez $A$. salmonicida pourrait également être due à sa provenance des eaux contaminées par les effluents hospitalier et autres déchets. une étude menée en Irlande a montré que les eaux de rivières prélevées à côté d'un point de versement d'effluents hospitalier contenaient plus de souches Aeromonas productrices de $\beta$-lactamases à spectre élargi et résistantes aux antibiotiques (Drieux-Rouzet and Jarlier 2014). Par ailleurs, les résultats de la souche d'E. coli du milieu hospitalier a montré une multirésistance aux céphalosporines de troisième génération peu importe la classe de ces dernières. Ces résultats avaient déjà été révélés dans bon nombre d'étude soulignant la multirésistance des $E$. coli aux $\mathrm{C}_{3} \mathrm{G}$ (Ferroni et al . 2003). Aussi, des nombreux auteurs ont démontré dans leurs travaux que les années 2000 ont été marquées par l'évolution mondiale des souches d'E. coli produisant un nouveau type de $\beta$-lactamase à spectres étendu (CTX-M) aussi bien dans les hôpitaux qu'au sein des communautés (Robin et al . 2012; Baba Ahmed-Kazi Tani and Arlet 2014). Effectivement, Mkaouar et al ont montré dans une étude la résistance des entérobactéries aux céphalosporines de troisième génération dans les hôpitaux de Sfax en Tunisie (Mkaouar et al . 2008). De plus, la prévalence de la résistance des $E$. coli aux céphalosporines de troisième génération augmenterait dans les hôpitaux et les données françaises et européennes montrent que cette résistance se diffuserait également dans les communautés (Andremont 1997.). La mise en évidence de la multirésistance des souches bactériennes isolées aussi bien de l'environnement que du milieu hospitalier a été faite dans les pays en voie de développement mais également dans les pays développés. Indéniablement, l'étude menée d'une part sur les bactéries isolées des eaux de Franceville et d'autre part sur la souche du milieu hospitalier ne pourrait que conforter ce phénomène de multirésistance. Enfin, les résultats du rapport a confirment la nature bactéricide des $C_{3} G$ testés. La large utilisation de ces molécules est principalement liée leur pouvoir bactéricide (Robin et al . 2012). 


\section{CONCLUSION}

II ressort de cette étude que le phénomène de multirésistance est sans aucun doute présent chez les bactéries isolées des eaux des lacs, de la rivière M'passa et du fleuve Ogooué de Franceville, car les germes isolés de ces eaux sont tous résistants à la cefpodoxime et à la céfixime. De plus, il se dégage un caractère de multirésistance aux quatre (4) $\mathrm{C}_{3} \mathrm{G}$ testées aussi bien pour Pseudomonas aeruginosa qu'Aeromonas salmonicida et la souche Escherichia coli du milieu hospitalier. Ainsi, la maitrise des bactéries multirésistantes des eaux reste un enjeu de santé publique dans notre pays car ces souches bactériennes

\section{RÉFÉRENCE}

Andremont $A$ (1997.). Définition de la multirésistance bactérienne. Réanimation Urgences 6 (2):175182. doi:http://dx.doi.org/10.1016/S1164$\underline{6756(97) 80078-3}$

Andremont A (2002) Pression de sélection antibiotique, flores commensales et évolution de la résistance. Journal de Pédiatrie et de $\begin{array}{lll}\text { Puériculture } \quad 15 & \text { (3):160-165. }\end{array}$ doi:http://dx.doi.org/10.1016/S09877983(02)83040-7

Baba Ahmed-Kazi Tani Z, Arlet G (2014) Actualité de la résistance aux antibiotiques chez les bacilles à Gram négatif en Algérie. Pathologie Biologie 62

(3):169-178. doi:http://dx.doi.org/10.1016/.patbio.2014.01.0 $\underline{05}$

Bush K, Jacoby GA, Medeiros AA (1995) A functional classification scheme for beta-lactamases and its correlation with molecular structure. Antimicrobial agents and chemotherapy 39 (6):1211-1233

Costanzo SD, Murby J, Bates J (2005) Ecosystem response to antibiotics entering the aquatic environment. Marine pollution bulletin 51 (14):218-223. doi:10.1016/j.marpolbul.2004.10.038

Drieux-Rouzet L, Jarlier V (2014) Bactéries multirésistantes dans l'eau : modéles des entérobactéries productrices de bètalactamase à spectreétendu. Revue Francophone des Laboratoires 2014 (460):7579

Ferroni A, Sermet-Gaudelus I, Abachin E, Quesnes G, Lenoir G, Berche P, Gaillard JL (2003) Caractéristiques phénotypiques et génotypiques des souches atypiques de sont à l'origine de nombreuses infections. Dans ce contexte, une prise en charge plus rigoureuse de l'environnement est plus que nécessaire. D'une part, l'amélioration des procédés de traitement des eaux usées et d'autre part la décontamination des effluents hospitaliers, industriels par des procédés physicochimiques et microbiologiques est donc essentiel. Aussi, la quantification et la caractérisation génotypique par recherche de gène de résistance permettraient d'évaluer les risques liés aux possibles transferts des gènes de résistance et infections.

bacilles à Gram négatif non fermentants isolées chez des patients atteints de mucoviscidose. Pathologie Biologie 51 (7):405-

411.doi:http://dx.doi.org/10.1016/S03698114(03)00021-X

Galvin S, Boyle F, Hickey $P$, Vellinga A, Morris D, Cormican M (2010) Enumeration and characterization of antimicrobial-resistant Escherichia coli bacteria in effluent from municipal, hospital, and secondary treatment facility sources. Applied and environmental microbiology 76 (14):4772-4779

Garcia-Armisen T, Anzil A, Cornelis P, Chevreuil M, Servais P (2013) Identification of antimicrobial resistant bacteria in rivers: insights into the cultivation bias. Water research 47 (14):49384947

Garcia-Armisen T, Thouvenin B, Servais P (2006) Modelling faecal coliforms dynamics in the Seine estuary, France. Water Sci Technol 54 (3):177-184

Garcia-Armisen T, Vercammen K, Passerat J, Triest D, Servais P, Cornelis P (2011) Antimicrobial resistance of heterotrophic bacteria in sewagecontaminated rivers. Water research 45 (2):788-796

Harris S, Morris C, Morris D, Cormican M, Cummins E (2014) Antimicrobial resistant Escherichia coli in the municipal wastewater system: Effect of hospital effluent and environmental fate. Science of The Total Environment 468 à 469 (0):1078-1085

Hijosa-Valsero M, Fink G, Schlusener MP, SidrachCardona R, Martin-Villacorta J, Ternes T, Becares $E$ (2011) Removal of antibiotics from 
urban wastewater by constructed wetland optimization. Chemosphere 83 (5):713-719. doi:10.1016/j.chemosphere.2011.02.004

Kulkarni HM, Nagaraj R, Jagannadham MV (2015) Protective role of $E$. coli outer membrane vesicles against antibiotics. Microbiological Research

181:1-7. doi:http://dx.doi.org/10.1016/..micres.2015.07. 008

Li XZ, Nikaido H (2009) Efflux-mediated drug resistance in bacteria: an update. Drugs 69 (12):15551623. doi:10.2165/11317030-00000000000000

Li XZ, Plesiat P, Nikaido H (2015) The challenge of efflux-mediated antibiotic resistance in Gramnegative bacteria. Clinical microbiology $\begin{array}{lll}\text { reviews } & 28 & \text { (2):337-418. }\end{array}$ doi:10.1128/CMR.00117-14

Lister PD, Wolter DJ, Hanson ND (2009) Antibacterialresistant Pseudomonas aeruginosa: clinical impact and complex regulation of chromosomally encoded resistance mechanisms. Clinical microbiology reviews 22 (4):582-610. doi:10.1128/CMR.00040-09

Mérens A, Delacour H, Plésiat $P$, Cavallo J-D, Jeannot $K$ (2011) Pseudomonas aeruginosa et résistance aux antibiotiques. Revue Francophone des Laboratoires 2011 (435):4962. doi:http://dx.doi.org/10.1016/S1773035X(11)71102-9

Mkaouar D, Mahjoubi F, Mezghani S, Znazen A, Ktari S, Hammami A (2008) Étude de la résistance des entérobactéries aux céphalosporines de troisième génération dans les hôpitaux de Sfax, Tunisie (1999-2005). Médecine et

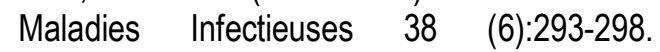
doi:http://dx.doi.org/10.1016/j.medmal.2007.11 .017

Nola M, Njine T, Sikati VF, Djuikom E (2001) « Distribution de Pseudomonas aeruginosa et Aeromonas hydrophila dans les eaux de la nappe phréatique superficielle en zone équatoriale au Cameroun et relations avec quelques paramètres chimiques du milieu. ". Journal of Water Science 14:35-53

Passerat J, Tamtam F, Lebot B, Eurin J, Chevreuil M, Servais $P$ (2010) Rejets hospitaliers d'antibiotiques et de bactéries fécales antibiorésistantes dans les rivières du bassin de la Seine. Eur $\mathrm{j}$ water qual:1-13

Philippon A, Arlet G, Jacoby GA (2002) Plasmiddetermined AmpC-type beta-lactamases. Antimicrobial agents and chemotherapy 46 (1):1-11

Prado T, Pereira WC, Silva DM, Seki LM, Carvalho AP, Asensi MD (2008) Detection of extendedspectrum beta-lactamase-producing Klebsiella pneumoniae in effluents and sludge of a hospital sewage treatment plant. Letters in applied microbiology 46 (1):136-141

Robin F, Gibold L, Bonnet R (2012) Résistances naturelles et acquises aux $\beta$-lactamines chez les entérobactéries : comment les identifier en pratique quotidienne ? Revue Francophone des Laboratoires 2012 (445):47-58. doi:http://dx.doi.org/10.1016/S1773035X(12)71676-3

Servais P, Passerat J (2009) Antimicrobial resistance of fecal bacteria in waters of the Seine river watershed (France). The Science of the total environment 408 (2):365-372

Sidrach-Cardona R, Bécares E (2013) Fecal indicator bacteria resistance to antibiotics in experimental constructed wetlands. Ecological Engineering 50:107-111. doi:http://dx.doi.org/10.1016/j.ecoleng.2012.01 .001

Sidrach-Cardona R, Hijosa-Valsero M, Marti E, Balcázar JL, Becares E (2014) Prevalence of antibiotic-resistant fecal bacteria in a river impacted by both an antibiotic production plant and urban treated discharges. Science of The Total Environment 488-489:220-227. doi:http://dx.doi.org/10.1016/j.scitotenv.2014.0 4.100

Stratton CW (1990) Outer membrane protein D2 catalyzes facilitated diffusion of carbapenems and penems through the outer membrane of Pseudomonas aeruginosa: Trias J, Nikaido $\mathrm{H}$ : Antimicrob Agents Chemother 34:52-57, 1990. Infectious Diseases Newsletter 9 (4):32. doi:http://dx.doi.org/10.1016/02782316(90)90078-T

Weiss K (2002) La résistance bactérienne : la nouvelle guerre froide. Le Médecin du Québec 37 (3):41-49 